\title{
On certain classes of bi-univalent functions related to m-fold symmetry
}

\author{
Saqib Hussain ${ }^{\mathrm{a}}$, Shahid Khan ${ }^{\mathrm{b}}$, Muhammad Asad Zaighum ${ }^{\mathrm{b}}$, Maslina Darus ${ }^{\mathrm{c}, *}$ \\ ${ }^{a}$ COMSATS Institute of Information Technology, Abbotabad, Pakistan. \\ ${ }^{b}$ Department of Mathematics, Riphah International University Islamabad, Pakistan. \\ ' School of Mathematical Sciences, Faculty of Science and Technology, Universiti Kebangsaan Malaysia 43600, Bangi, Selangor, \\ Malaysia.
}

Communicated by $\mathrm{Y}$. Hu

\begin{abstract}
In our present investigation, we introduce two new subclasses $S_{\Sigma_{m}}(\alpha, \lambda, \mu)$ and $S_{\Sigma_{m}}(\beta, \lambda, \mu)$ of analytic and m-fold symmetric bi-univalent functions in the open unit disk E. Results concerning coefficient estimates for the functions of these classes are derived. Many interesting new and already existing corollaries are also presented.
\end{abstract}

Keywords: m-Fold symmetry, bi-univalent functions, coefficient estimates.

2010 MSC: $30 C 45,30 \mathrm{C} 50$.

(C)2018 All rights reserved.

\section{Introduction}

Let $\mathcal{A}$ denotes the class of all functions $\mathrm{f}(z)$ which are analytic in the open unit disk $E=\{z:|z|<1\}$ and has the Taylor series expansion of the form:

$$
f(z)=z+\sum_{n=2}^{\infty} a_{n} z^{n} .
$$

A function is said to be univalent if it never takes the same value twice. By $\mathcal{S}$ we mean the subclass of $\mathcal{A}$ consisting of univalent functions. Every univalent function $f \in \mathcal{S}$ has an inverse $\mathrm{f}^{-1}$ which is defined as:

$$
\mathrm{f}^{-1}(\mathrm{f}(z))=z, \quad z \in \mathrm{E},
$$

\footnotetext{
*Corresponding author

Email addresses: saqib_math@yahoo.com (Saqib Hussain), shahidmath761@gmail.com (Shahid Khan), asadzaighum@gmail.com (Muhammad Asad Zaighum), maslina@ukm.edu.my (Maslina Darus)
}

doi: $10.22436 /$ jnsa.011.04.06

Received: 2017-09-19 Revised: 2017-12-01 Accepted: 2017-12-06 
and

$$
f\left(f^{-1}(w)\right)=w,|w|<r_{0}(f), r_{0}(f) \geqslant \frac{1}{4}
$$

where

$$
g_{1}(w)=f^{-1}(w)=w-a_{2} w^{2}+\left(2 a_{2}^{2}-a_{3}\right) w^{3}-\left(5 a_{2}^{3}-5 a_{2} a_{3}+a_{4}\right) w^{4}+\cdots .
$$

A function $\mathrm{f} \in \mathcal{A}$ is said to be bi-univalent in $\mathrm{E}$ if both $\mathrm{f}$ and $\mathrm{f}^{-1}$ are univalent in $\mathrm{E}$. Let $\Sigma$ denotes the class of analytic and bi-univalent functions in E. Few examples of functions in class $\Sigma$ are

$$
h_{1}(z)=\frac{z}{1-z}, h_{2}(z)=-\log (1-z), h_{3}(z)=\frac{1}{2} \log \left(\frac{1+z}{1-z}\right), z \in E
$$

For $\mathrm{f} \in \Sigma$, Lewin [20], showed that $\left|\mathrm{a}_{2}\right|<1.5$. For more work on bi-univalent one can refer to [1, 3$13,15,16,18,19,21-26,28-31]$.

Let $m$ be a positive integer. A domain $E$ is said to be $m$-fold symmetric if

$$
f\left(e^{i \frac{2 \pi}{m}} z\right)=e^{i \frac{2 \pi}{m}} f(z), z \in E, f \in \mathcal{A} .
$$

For each function $f \in \mathcal{S}$, the function

$$
h(z)=\sqrt[m]{f\left(z^{m}\right)}
$$

is univalent and maps the unit disk $E$ into a region with $m$-fold symmetry.

We denote by $\mathcal{S}^{m}$ the class of $m$-fold symmetric univalent functions in $E$ and clearly $S^{1}=S$. Let $f \in \mathcal{S}^{m}$ has a series expansion of the form

$$
f(z)=z+\sum_{k=1}^{\infty} a_{m k+1} z^{m k+1} .
$$

Srivastava et al. [25], introduced a natural extensions of $m$-fold symmetric univalent functions and defined the class $\Sigma_{m}$ of symmetric bi-univalent functions. They obtained the series expansion for $g=\mathrm{f}^{-1}$ as:

$$
g(w)=f^{-1}(w)=\left\{\begin{array}{l}
w-a_{m+1} w^{m+1}+\left((m+1) a_{m+1}^{2}-a_{2 m+1}\right) w^{2 m+1}, \\
-\left\{\begin{array}{c}
\frac{1}{2}(m+1)(3 m+2) a_{m+1}^{3} \\
\left.-(3 m+2) a_{m+1} a_{2 m+1}+a_{3 m+1}\right)
\end{array}\right\} w^{3 m+1}+\cdots,
\end{array}\right.
$$

where $f$ is given by (1.2). For $m=1$, the equation (1.3) coincides with the equation (1.1) of the class $\Sigma$. Under the following assumptions, $z, w \in E, f^{-1}=g, m \in N, 0<\alpha \leqslant 1,0 \leqslant \beta<1,0 \leqslant \mu$, and $0 \leqslant \lambda \leqslant 1$, we introduced new subclasses of $m$-fold symmetric bi-univalent functions and derive initial coefficient bounds for $\left|a_{m+1}\right|$ and $\left|a_{2 m+1}\right|$ for these classes.

1.1. The class $\mathcal{S}_{\Sigma_{m}}(\alpha, \lambda, \mu)$

Definition 1.1. A function $f \in \Sigma_{m}$, is said to be in class $\mathcal{S}_{\Sigma_{m}}(\alpha, \lambda, \mu)$ if the following conditions are satisfied

$$
\left|\arg \left[(1-\lambda)\left(\frac{z^{1-\mu} f^{\prime}(z)}{[f(z)]^{1-\mu}}\right)+\lambda\left(1+\frac{z^{2-\mu} f^{\prime \prime}(z)}{\left[z f^{\prime}(z)\right]^{1-\mu}}\right)\right]\right|<\frac{\alpha \pi}{2},
$$

and

$$
\left|\arg \left[(1-\lambda)\left(\frac{w^{1-\mu} g^{\prime}(w)}{[g(w)]^{1-\mu}}\right)+\lambda\left(1+\frac{w^{2-\mu} g^{\prime \prime}(w)}{\left[w g^{\prime}(w)\right]^{1-\mu}}\right)\right]\right|<\frac{\alpha \pi}{2} .
$$

Remark 1.2. On specializing the parameter $\lambda, \mu, m$ one can state the various new as well as known subclasses of analytic bi-univalent functions studied earlier in the literature. 
(i) For $\mathrm{m}=1$, we obtain new class of bi-univalent function.

$$
\mathcal{S}_{\Sigma_{m}}(\alpha, \lambda, \mu)=\mathcal{S}_{\Sigma}(\alpha, \lambda, \mu)
$$

(ii) For $\lambda=0$, we obtain new class which consists $m$-fold symmetric bi starlike function.

$$
\mathcal{S}_{\Sigma_{m}}(\alpha, \lambda, \mu)=\mathcal{R}_{\Sigma_{m}}(\alpha, \mu)
$$

(iii) For $\lambda=1$, we obtain a new class which consists $m$-fold symmetric convex bi-univalent function.

$$
\mathcal{S}_{\Sigma_{m}}(\alpha, \lambda, \mu)=\mathcal{C}_{\Sigma_{m}}(\alpha, \mu)
$$

(iv) For $\lambda=0$, and $\mu=0$, we obtain class which consists $m$-fold symmetric bi-univalent function [2].

$$
\mathcal{S}_{\Sigma_{m}}(\alpha, \lambda, \mu)=\mathcal{S}_{\Sigma, m}^{\alpha}
$$

(v) For $\lambda=0, \mathrm{~m}=1$ and $\mu=0$, we obtain class of bi-univalent function introduced by Brannan and Taha [5].

$$
\mathcal{S}_{\Sigma_{m}}(\alpha, \lambda, \mu)=\mathcal{S}_{\Sigma}^{*}(\alpha) .
$$

(vi) For $\lambda=0$ and $\mu=1$, we obtain class which consists $m$-fold symmetric bi-univalent function introduced by Srivastava et al. [27].

$$
\mathcal{S}_{\Sigma_{\mathrm{m}}}(\alpha, \lambda, \mu)=\mathcal{H}_{\Sigma, m}(\alpha) \text {. }
$$

(vii) For $\lambda=0, m=1$, and $\mu=1$, we obtain class of bi-univalent function introduced by Srivastava et al. [26].

$$
\mathcal{S}_{\Sigma_{\mathfrak{m}}}(\alpha, \lambda, \mu)=\mathcal{H}_{\Sigma}(\alpha)
$$

1.2. The class $\mathcal{S}_{\Sigma_{m}}(\beta, \lambda, \mu)$

Definition 1.3. A function $f \in \Sigma_{m}$ is said to be in class $S_{\Sigma_{m}}(\beta, \lambda, \mu)$ if the following conditions are satisfied

$$
\operatorname{Re}\left[(1-\lambda)\left(\frac{z^{1-\mu} f^{\prime}(z)}{[f(z)]^{1-\mu}}\right)+\lambda\left(1+\frac{z^{2-\mu} f^{\prime \prime}(z)}{\left[z f^{\prime}(z)\right]^{1-\mu}}\right)\right]>\beta,
$$

and

$$
\operatorname{Re}\left[(1-\lambda)\left(\frac{w^{1-\mu} g^{\prime}(w)}{[g(w)]^{1-\mu}}\right)+\lambda\left(1+\frac{w^{2-\mu} g^{\prime \prime}(w)}{\left[w g^{\prime}(w)\right]^{1-\mu}}\right)\right]>\beta
$$

Remark 1.4. On specializing the parameters $\lambda, \mu, \mathrm{m}$ one can state the various new as well as known subclasses of analytic bi-univalent functions studied earlier in the literature.

(i) For $m=1$, we obtain new class of bi-univalent function.

$$
\mathcal{S}_{\Sigma_{m}}(\beta, \lambda, \mu)=\mathcal{S}_{\Sigma}(\beta, \lambda, \mu)
$$

(ii) For $\lambda=0$, we obtain new class which consists $m$-fold symmetric bi starlike function.

$$
\mathcal{S}_{\Sigma_{m}}(\beta, \lambda, \mu)=\mathcal{R}_{\Sigma_{m}}(\beta, \mu) .
$$

(iii) For $\lambda=1$, we obtain new class which consists $m$-fold symmetric convex bi-univalent function.

$$
\mathcal{S}_{\Sigma_{m}}(\beta, \lambda, \mu)=\mathcal{C}_{\Sigma_{m}}(\beta, \mu) .
$$

(iv) For $\lambda=0$, and $\mu=0$, we obtain class which consists $m$-fold symmetric bi-univalent function [17].

$$
\mathcal{S}_{\Sigma_{m}}(\beta, \lambda, \mu)=\mathcal{N}_{\Sigma, m}^{0}(\beta, 1) .
$$


(v) For $\lambda=0, m=1$ and $\mu=0$, we obtain class of bi-univalent function introduced by Brannan and Taha [5].

$$
\mathcal{S}_{\Sigma_{m}}(\beta, \lambda, \mu)=\mathcal{S}_{\Sigma}^{*}(\beta) .
$$

(vi) For $\lambda=0$ and $\mu=1$, we obtain class which consists $m$-fold symmetric bi-univalent function introduced by Srivastava et al. [27].

$$
\mathcal{S}_{\Sigma_{m}}(\beta, \lambda, \mu)=\mathcal{H}_{\Sigma, m}(\beta) .
$$

(vii) For $\lambda=0, m=1$, and $\mu=1$, we obtain of bi-univalent function introduced by Srivastava et al. [26]

$$
\mathcal{S}_{\Sigma_{\mathfrak{m}}}(\beta, \lambda, \mu)=\mathcal{H}_{\Sigma}(\beta) .
$$

In order to establish our main results, we shall required the following lemma.

Lemma 1.5 ([14]). $p \in P,\left|c_{n}\right| \leqslant 2, n \in N$, where the Caratheodory class $P$ is the family of all functions $p$ analytic in $\mathrm{E}$ for which $\operatorname{Re}(\mathrm{p}(z))>0$,

$$
p(z)=1+c_{1} z+c_{2} z^{2}+\cdots .
$$

\section{Main results}

Theorem 2.1. Let $f$ given by (1.2) is in the class $\mathcal{S}_{\Sigma_{m}}(\alpha, \lambda, \mu)$, then

$$
\left|a_{m+1}\right| \leqslant \frac{2 \alpha}{\sqrt{\alpha Q_{1}(\lambda, \mu, m)-\left(\alpha Q_{2}(\lambda, \mu, m)+Q_{3}(\lambda, \mu, m)\right)}}
$$

and

$$
\left|a_{2 m+1}\right| \leqslant \frac{2 \alpha}{\left[(2 m+\mu)+\lambda\left(4 m^{2}-\mu\right)\right]}+\frac{2 \alpha^{2}\left[Q_{1}(\lambda, \mu, m)+Q_{2}(\lambda, \mu, m)\right]}{\left[(2 m+\mu)+\lambda\left(4 m^{2}-\mu\right)\right]\left[(m+\mu)+\lambda\left(m^{2}-\mu\right)\right]^{2}},
$$

where $\mathrm{Q}_{1}(\lambda, \mu, \mathrm{m}), \mathrm{Q}_{2}(\lambda, \mu, \mathrm{m}), \mathrm{Q}_{3}(\lambda, \mu, \mathrm{m})$ are given by (2.9), (2.10), (2.12), respectively.

Proof. Let $f \in \mathcal{S}_{\Sigma_{m}}(\alpha, \lambda, \mu)$, then

$$
(1-\lambda)\left(\frac{z^{1-\mu} f^{\prime}(z)}{[f(z)]^{1-\mu}}\right)+\lambda\left(1+\frac{z^{2-\mu} f^{\prime \prime}(z)}{\left[z f^{\prime}(z)\right]^{1-\mu}}\right)=[p(z)]^{\alpha},
$$

and for its inverse map $g=f^{-1}$, we have

$$
(1-\lambda)\left(\frac{w^{1-\mu} g^{\prime}(w)}{[g(w)]^{1-\mu}}\right)+\lambda\left(1+\frac{w^{2-\mu} g^{\prime \prime}(w)}{\left[w g^{\prime}(w)\right]^{1-\mu}}\right)=[q(w)]^{\alpha},
$$

where $p(z)$ and $q(w)$ have the following forms:

$$
p(z)=1+p_{m} z^{m}+p_{2 m} z^{2 m}+\cdots,
$$

and

$$
\mathrm{q}(w)=1+\mathrm{q}_{\mathrm{m}} w^{\mathrm{m}}+\mathrm{q}_{2 \mathrm{~m}} w^{2 \mathrm{~m}}+\cdots .
$$

Now equating the coefficients in (2.1) and (2.2) we obtain

$$
\begin{aligned}
& (m+\mu)+\lambda\left(m^{2}-\mu\right) a_{m+1}=\alpha p_{m}, \\
& \left\{\begin{array}{c}
(2 m+\mu)+\lambda\left(4 m^{2}-\mu\right) a_{2 m+1} \\
-Q_{2}(\lambda, \mu, m) a_{m+1}^{2}
\end{array}\right\}=\alpha p_{2 m}+\frac{\alpha(\alpha-1)}{2} p_{m}^{2}, \\
& -(m+\mu)+\lambda\left(m^{2}-\mu\right) a_{m+1}=\alpha q_{m} \text {, }
\end{aligned}
$$




$$
\left\{\begin{array}{c}
Q_{1}(\lambda, \mu, m) a_{m+1}^{2} \\
-(2 m+\mu)+\lambda\left(4 m^{2}-\mu\right) a_{2 m+1}
\end{array}\right\}=\alpha q_{2 m}+\frac{\alpha(\alpha-1)}{2} q_{m}^{2} .
$$

From (2.3) and (2.5) we obtain

$$
p_{m}=-q_{m}
$$

and

$$
\left\{(m+\mu)+\lambda\left(m^{2}-\mu\right)\right\}^{2} 2 a_{m+1}^{2}=\alpha^{2}\left(p_{m}^{2}+q_{m}^{2}\right) .
$$

Also form (2.4), (2.6), and (2.8) we have

$$
\begin{aligned}
\left\{Q_{1}(\lambda, \mu, m)-Q_{2}(\lambda, \mu, m)\right\} a_{m+1}^{2} & =\alpha\left(p_{2 m}+q_{2 m}\right)+\frac{\alpha(\alpha-1)}{2}\left(p_{m}^{2}+q_{m}^{2}\right) \\
& =\left\{\begin{array}{c}
\alpha\left(p_{2 m}+q_{2 m}\right) \\
+\frac{(\alpha-1)}{\alpha}\left\{(m+\mu)+\lambda\left(m^{2}-\mu\right)\right\}^{2} a_{m+1}^{2}
\end{array}\right\},
\end{aligned}
$$

where

$$
\begin{aligned}
& \left.\mathrm{Q}_{1}(\lambda, \mu, \mathrm{m})=[(1-\lambda)(1+2 \mathrm{~m})(\mathrm{m}+\mu)+\lambda \mathrm{m}(\mathrm{m}+1)\{3 \mathrm{~m}+\mu(\mathrm{m}+1)+1)\}\right], \\
& \mathrm{Q}_{2}(\lambda, \mu, \mathrm{m})=\frac{(1-\mu)}{2 !}\left\{(1-\lambda)(\mu+2 \mathrm{~m})+2 \lambda \mathrm{m}(\mathrm{m}+1)^{2}\right\} .
\end{aligned}
$$

Therefore, we have

$$
a_{m+1}^{2}=\frac{\alpha^{2}\left(p_{2 m}+q_{2 m}\right)}{\alpha Q_{1}(\lambda, \mu, m)-\left[\alpha Q_{2}(\lambda, \mu, m)+Q_{3}(\lambda, \mu, m)\right]}
$$

where

$$
\mathrm{Q}_{3}(\lambda, \mu, \mathrm{m})=(\alpha-1)\left\{(\mathrm{m}+\mu)+\lambda\left(\mathrm{m}^{2}-\mu\right)\right\}^{2} .
$$

Applying Lemma 1.5, on equation (2.11) for the coefficients $p_{2 m}$ and $q_{2 m}$, we obtain

$$
\left|a_{m+1}\right| \leqslant \frac{2 \alpha}{\sqrt{\alpha Q_{1}(\lambda, \mu, m)-\left[\alpha Q_{2}(\lambda, \mu, m)+Q_{3}(\lambda, \mu, m)\right]}} .
$$

Next, in order to find the bound on $\left|a_{2 m+1}\right|$, by subtracting (2.6) from (2.4), we obtain

$$
\left[\begin{array}{c}
2\left\{(2 m+\mu)+\lambda\left(4 m^{2}-\mu\right)\right\} a_{2 m+1} \\
-\left\{Q_{2}(\lambda, \mu, m)+Q_{1}(\lambda, \mu, m)\right\} a_{m+1}^{2}
\end{array}\right]=\alpha\left(p_{2 m}-q_{2 m}\right)+\frac{\alpha(\alpha-1)}{2}\left(p_{m}^{2}-q_{m}^{2}\right) .
$$

Then, in view of (2.7) and (2.8), and applying Lemma 1.5, on (2.13) for the coefficients $p_{2 m}, q_{2 m}, p_{m}$ and $q_{m}$ we have

$$
\left|a_{2 m+1}\right| \leqslant \frac{2 \alpha}{\left[(2 m+\mu)+\lambda\left(4 m^{2}-\mu\right)\right]}+\frac{2 \alpha^{2}\left[Q_{1}(\lambda, \mu, m)+Q_{2}(\lambda, \mu, m)\right]}{\left[(2 m+\mu)+\lambda\left(4 m^{2}-\mu\right)\right]\left[(m+\mu)+\lambda\left(m^{2}-\mu\right)\right]^{2}},
$$

which completes the proof of Theorem 2.1.

For $\mathrm{m}=1$, in Theorem 2.1, we have the following Corollary.

Corollary 2.2. Let $f$ given by (1.2) is in the class $\mathcal{S}_{\Sigma}(\alpha, \lambda, \mu)$, then

$$
\left|a_{2}\right| \leqslant \frac{2 \alpha}{\sqrt{\alpha Q_{10}(\lambda, \mu)-\left(\alpha Q_{11}(\lambda, \mu)+Q_{12}(\lambda, \mu)\right)}},
$$

and

$$
\left|a_{3}\right| \leqslant \frac{2 \alpha}{(2+\mu)+\lambda(4-\mu)}+\frac{2 \alpha^{2}\left[Q_{10}(\lambda, \mu)+Q_{11}(\lambda, \mu)\right]}{[(2+\mu)+\lambda(4-\mu)][(1+\mu)+\lambda(1-\mu)]^{2}}
$$


where

$$
\begin{aligned}
& \mathrm{Q}_{10}(\lambda, \mu)=3(1-\lambda)(1+\mu)+4 \lambda(2+\mu), \\
& \mathrm{Q}_{11}(\lambda, \mu)=\frac{(1-\mu)}{2 !}\{(1-\lambda)(\mu+2)+8 \lambda\}, \\
& \mathrm{Q}_{12}(\lambda, \mu)=(\alpha-1)\{(1+\mu)+\lambda(1-\mu)\}^{2} .
\end{aligned}
$$

For $\lambda=0$ in Theorem 2.1, we have the following Corollary.

Corollary 2.3. Let $f$ given by (1.2) is in the class $\mathcal{R}_{\Sigma_{m}}(\alpha, \mu)$, then

$$
\left|a_{m+1}\right| \leqslant \frac{2 \alpha}{\sqrt{\alpha Q_{4}(\mu, m)-\left(\alpha Q_{5}(\mu, m)+Q_{6}(\mu, m)\right)}},
$$

and

$$
\left|a_{2 m+1}\right| \leqslant \frac{2 \alpha}{(2 m+\mu)}+\frac{2 \alpha^{2}\left[Q_{4}(\mu, m)+Q_{5}(\mu, m)\right]}{(2 m+\mu)(m+\mu)^{2}},
$$

where

$$
\begin{aligned}
& \mathrm{Q}_{4}(\mu, \mathrm{m})=(1+2 \mathrm{~m})(\mathrm{m}+\mu), \\
& \mathrm{Q}_{5}(\mu, \mathrm{m})=\frac{(1-\mu)}{2 !}(\mu+2 \mathrm{~m}), \\
& \mathrm{Q}_{6}(\mu, \mathrm{m})=(\alpha-1)(\mathrm{m}+\mu)^{2} .
\end{aligned}
$$

For $\lambda=1$ in Theorem 2.1 we have the following Corollary.

Corollary 2.4. Let $f$ given by (1.2) is in the class $\mathcal{C}_{\Sigma_{m}}(\alpha, \mu)$, then

$$
\left|a_{m+1}\right| \leqslant \frac{2 \alpha}{\sqrt{\alpha Q_{7}(\mu, m)-\left(\alpha Q_{8}(\mu, m)+Q_{9}(\mu, m)\right)}},
$$

and

$$
\left|a_{2 m+1}\right| \leqslant \frac{2 \alpha}{\left[(2 m+\mu)+\left(4 m^{2}-\mu\right)\right]}+\frac{2 \alpha^{2}\left[Q_{7}(\mu, m)+Q_{8}(\mu, m)\right]}{\left[(2 m+\mu)+\left(4 m^{2}-\mu\right)\right]\left[(m+\mu)+\left(m^{2}-\mu\right)\right]^{2}},
$$

where

$$
\begin{aligned}
& \left.\mathrm{Q}_{7}(\mu, \mathrm{m})=\mathrm{m}(\mathrm{m}+1)\{3 \mathrm{~m}+\mu(\mathrm{m}+1)+1)\right\}, \\
& \mathrm{Q}_{8}(\mu, \mathrm{m})=\frac{(1-\mu)}{2 !}\left\{2 \mathrm{~m}(\mathrm{~m}+1)^{2}\right\}, \\
& \mathrm{Q}_{9}(\mu, \mathrm{m})=(\alpha-1)\left\{(\mathrm{m}+\mu)+\left(\mathrm{m}^{2}-\mu\right)\right\}^{2} .
\end{aligned}
$$

For $\lambda=0$ and $\mu=1$ in Theorem 2.1, we obtain the following Corollary.

Corollary 2.5 ([27]). Let $\mathrm{f}$ given by (1.2) be in the class $\mathcal{H}_{\Sigma, \mathrm{m}}^{\alpha}$, then

$$
\left|a_{m+1}\right| \leqslant \frac{2 \alpha}{\sqrt{(1+m)(1+m+2 \alpha)}}, \quad \text { and } \quad\left|a_{2 m+1}\right| \leqslant \frac{2 \alpha}{(2 m+1)}+\frac{2 \alpha^{2}}{(m+1)} .
$$

For $\lambda=0, m=1$, and $\mu=1$, in Theorem 2.1, we obtain the following Corollary.

Corollary 2.6 ([26]). Let $\mathrm{f}$ given by (1.2) be in the class $\mathrm{H}_{\Sigma}^{\alpha}$, then

$$
\left|\mathrm{a}_{2}\right| \leqslant \frac{\alpha}{\sqrt{(1+\alpha)}}, \text { and }\left|\mathrm{a}_{3}\right| \leqslant \frac{2 \alpha}{3}+\frac{\alpha^{2}}{1} .
$$


For $\lambda=0$ and $\mu=0$ in Theorem 2.1, we obtain the following Corollary.

Corollary 2.7 ([2]). Let $\mathrm{f}$ given by (1.2) be in the class $\mathcal{S}_{\Sigma, \mathrm{m}}^{\alpha}$, then

$$
\left|a_{m+1}\right| \leqslant \frac{2 \alpha}{m \sqrt{\alpha+1}}, \quad \text { and } \quad\left|a_{2 m+1}\right| \leqslant \frac{\alpha}{m}+\frac{2 \alpha^{2}[(1+m)]}{m^{2}} .
$$

For $\lambda=0, m=1$, and $\mu=0$, in Theorem 2.1, we obtain the following Corollary.

Corollary 2.8 ([2]). Let $\mathrm{f}$ given by (1.2) be in the class $\mathcal{S}_{\Sigma}^{*}(\alpha)$, then

$$
\left|\mathrm{a}_{2}\right| \leqslant \frac{2 \alpha}{\sqrt{\alpha+1}}, \text { and }\left|\mathrm{a}_{3}\right| \leqslant 4 \alpha^{2}+\alpha .
$$

Theorem 2.9. Let $\mathrm{f}$ given by (1.2) is in the class $\mathcal{S}_{\Sigma_{m}}(\beta, \lambda, \mu)$, then

$$
\left|a_{m+1}\right| \leqslant \frac{2 \sqrt{(1-\beta)}}{\sqrt{\left\{Q_{1}(\lambda, \mu, m)-Q_{2}(\lambda, \mu, m)\right.}},
$$

and

$$
\left|a_{2 m+1}\right| \leqslant \frac{2(1-\beta)}{\left\{(2 m+\mu)+\lambda\left(4 m^{2}-\mu\right)\right\}}+\frac{2\left\{Q_{1}(\lambda, \mu, m)+Q_{2}(\lambda, \mu, m)\right\}(1-\beta)^{2}}{\left\{(2 m+\mu)+\lambda\left(4 m^{2}-\mu\right)\right\}\left\{(m+\mu)+\lambda\left(m^{2}-\mu\right)\right\}^{2}},
$$

where $\mathrm{Q}_{1}(\lambda, \mu, \mathrm{m})$ and $\mathrm{Q}_{2}(\lambda, \mu, \mathrm{m})$ are given by (2.9) and (2.10), respectively.

Proof. Let $f \in \mathcal{S}_{\Sigma_{m}}(\beta, \lambda, \mu)$, then

$$
(1-\lambda)\left(\frac{z^{1-\mu} f^{\prime}(z)}{[f(z)]^{1-\mu}}\right)+\lambda\left(1+\frac{z^{2-\mu} f^{\prime \prime}(z)}{\left[z f^{\prime}(z)\right]^{1-\mu}}\right)=\beta+(1-\beta) p(z),
$$

and for its inverse map $g=f^{-1}$, we have

$$
(1-\lambda)\left(\frac{w^{1-\mu} g^{\prime}(w)}{[g(w)]^{1-\mu}}\right)+\lambda\left(1+\frac{w^{2-\mu} g^{\prime \prime}(w)}{\left[w g^{\prime}(w)\right]^{1-\mu}}\right)=\beta+(1-\beta) q(w),
$$

where $p, q \in P$ and $g=f^{-1}$. Now, equating the coefficients in (2.20) and (2.21), we obtain

$$
\begin{aligned}
& (m+\mu)+\lambda\left(m^{2}-\mu\right) a_{m+1}=(1-\beta) p_{m}, \\
& \left\{(2 m+\mu)+\lambda\left(4 m^{2}-\mu\right)\right\} a_{2 m+1}-Q_{2}(\lambda, \mu, m) a_{m+1}^{2}=(1-\beta) p_{2 m}, \\
& -(m+\mu)+\lambda\left(m^{2}-\mu\right) a_{m+1}=(1-\beta) q_{m}, \\
& Q_{1}(\lambda, \mu, m) a_{m+1}^{2}-\left\{(2 m+\mu)+\lambda\left(4 m^{2}-\mu\right)\right\} a_{2 m+1}=(1-\beta) q_{2 m} .
\end{aligned}
$$

From (2.22) and (2.24) we obtain

$$
p_{m}=-q_{m}
$$

and

$$
2\left\{(m+\mu)+\lambda\left(m^{2}-\mu\right)\right\}^{2} a_{m+1}^{2}=(1-\beta)^{2}\left(p_{m}^{2}+q_{m}^{2}\right) .
$$

Adding (2.23) and (2.25), we have

$$
\left\{Q_{1}(\lambda, \mu, m)-Q_{2}(\lambda, \mu, m)\right\} a_{m+1}^{2}=(1-\beta)\left(p_{2 m}+q_{2 m}\right),
$$

therefore, we have

$$
a_{m+1}^{2}=\frac{(1-\beta)\left(p_{2 m}+q_{2 m}\right)}{Q_{1}(\lambda, \mu, m)-Q_{2}(\lambda, \mu, m)}
$$


Applying Lemma 1.5, on equation (2.28) for the coefficients $p_{2 m}$ and $q_{2 m}$, we obtain

$$
\left|a_{m+1}\right| \leqslant \frac{2 \sqrt{(1-\beta)}}{\sqrt{Q_{1}(\lambda, \mu, m)-Q_{2}(\lambda, \mu, m)}} .
$$

Next, in order to find the bound on $\left|a_{2 m+1}\right|$, by subtracting (2.25) from (2.23), we obtain

$$
\left[\begin{array}{c}
2\left\{(2 m+\mu)+\lambda\left(4 m^{2}-\mu\right)\right\} a_{2 m+1} \\
-\left\{Q_{1}(\lambda, \mu, m)+Q_{2}(\lambda, \mu, m)\right\} a_{m+1}^{2}
\end{array}\right]=(1-\beta)\left(p_{2 m}-q_{2 m}\right),
$$

then, in view of (2.26) and (2.27), and applying Lemma 1.5, on equation (2.29) for the coefficients $p_{2 m}$, $\mathrm{q}_{2 \mathrm{~m}}, \mathrm{p}_{\mathrm{m}}$, and $\mathrm{q}_{\mathrm{m}}$ we have

$$
\left|a_{2 m+1}\right| \leqslant \frac{2(1-\beta)}{(2 m+\mu)+\lambda\left(4 m^{2}-\mu\right)}+\frac{2\left\{Q_{1}(\lambda, \mu, m)+Q_{2}(\lambda, \mu, m)\right\}(1-\beta)^{2}}{\left\{(2 m+\mu)+\lambda\left(4 m^{2}-\mu\right)\right\}\left\{(m+\mu)+\lambda\left(m^{2}-\mu\right)\right\}^{2}},
$$

which completes the proof of Theorem 2.9.

For $\mathrm{m}=1$ in Theorem 2.9, we have the following Corollary.

Corollary 2.10. Let $f$ given by $(1.2)$ is in the class $\mathcal{S}_{\Sigma}(\beta, \lambda, \mu)$, then

$$
\left|a_{2}\right| \leqslant \frac{2 \sqrt{(1-\beta)}}{\sqrt{\left\{Q_{10}(\lambda, \mu)-Q_{11}(\lambda, \mu)\right\}}}
$$

and

$$
\left|\mathrm{a}_{3}\right| \leqslant \frac{2(1-\beta)}{\{(2+\mu)+\lambda(4-\mu)\}}+\frac{2\left\{\mathrm{Q}_{10}(\lambda, \mu)+\mathrm{Q}_{11}(\lambda, \mu)\right\}(1-\beta)^{2}}{\{(2+\mu)+\lambda(4-\mu)\}\{(1+\mu)+\lambda(1-\mu)\}^{2}},
$$

where $\mathrm{Q}_{10}(\lambda, \mu)$ and $\mathrm{Q}_{11}(\lambda, \mu)$ are given by (2.14) and (2.15).

For $\lambda=0$, in Theorem 2.9, we have the following Corollary.

Corollary 2.11. Let $f$ given by (1.2) is in the class $\mathcal{R}_{\Sigma_{m}}(\beta, \mu)$, then

$$
\left|a_{m+1}\right| \leqslant \frac{2 \sqrt{(1-\beta)}}{\sqrt{Q_{4}(\mu, m)-Q_{5}(\mu, m)}}
$$

and

$$
\left|a_{2 m+1}\right| \leqslant \frac{2(1-\beta)}{(2 m+\mu)}+\frac{2\left\{Q_{4}(\mu, m)+Q_{5}(\mu, m)\right\}(1-\beta)^{2}}{(2 m+\mu)(m+\mu)^{2}},
$$

where $\mathrm{Q}_{4}(\mu, \mathrm{m})$ and $\mathrm{Q}_{5}(\mu, \mathrm{m})$ are given by (2.16) and (2.17), respectively.

For $\lambda=1$, in Theorem 2.9, we have the following Corollary.

Corollary 2.12. Let $f$ given by $(1.2)$ is in the class $\mathcal{C}_{\Sigma_{m}}(\beta, \mu)$, then

$$
\left|a_{m+1}\right| \leqslant \frac{2 \sqrt{(1-\beta)}}{\sqrt{Q_{7}(\mu, m)-Q_{8}(\mu, m)}}
$$

and

$$
\left|a_{2 m+1}\right| \leqslant \frac{2(1-\beta)}{\left\{(2 m+\mu)+\left(4 m^{2}-\mu\right)\right\}}+\frac{2\left\{Q_{7}(\mu, m)+Q_{8}(\mu, m)\right\}(1-\beta)^{2}}{\left\{(2 m+\mu)+\left(4 m^{2}-\mu\right)\right\}\left\{(m+\mu)+\left(m^{2}-\mu\right)\right\}^{2}},
$$

where $\mathrm{Q}_{7}(\mu, \mathrm{m})$ and $\mathrm{Q}_{8}(\mu, \mathrm{m})$ are given by (2.18) and (2.19), respectively. 
For $\lambda=0$ and $\mu=1$ in Theorem 2.9, we obtain the following Corollary.

Corollary 2.13 ([27]). Let $\mathrm{f}$ given by (1.2) be in the class $\mathcal{H}_{\Sigma, \mathrm{m}}^{\beta}$, then

$$
\left|a_{m+1}\right| \leqslant \frac{2 \sqrt{(1-\beta)}}{\sqrt{(1+2 m)(m+1)}}, \quad \text { and } \quad\left|a_{2 m+1}\right| \leqslant \frac{2(1-\beta)}{(2 m+1)}+\frac{2(1-\beta)^{2}}{(m+1)} .
$$

For $\lambda=0, m=1$, and $\mu=1$, in Theorem 2.9, we obtain the following Corollary.

Corollary 2.14 ([26]). Let $\mathrm{f}$ given by (1.2) be in the class $\mathcal{H}_{\Sigma}^{\beta}$, then

$$
\left|a_{2}\right| \leqslant \sqrt{\frac{2(1-\beta)}{3}} \text {, and }\left|a_{3}\right| \leqslant \frac{2(1-\beta)}{3}+\frac{(1-\beta)^{2}}{1} .
$$

For $\lambda=0$ and $\mu=0$ in Theorem 2.9, we obtain the following Corollary.

Corollary 2.15 ([2]). Let $\mathrm{f}$ given by (1.2) be in the class $\mathcal{S}_{\Sigma_{\mathrm{m}}}^{\beta}$, then

$$
\left|a_{m+1}\right| \leqslant \sqrt{\frac{2(1-\beta)}{m}} \text {, and }\left|a_{2 m+1}\right| \leqslant \frac{(1-\beta)}{m}+\frac{2(1+m)(1-\beta)^{2}}{m^{2}} .
$$

For $\lambda=0, m=1$, and $\mu=0$, in Theorem 2.9, we obtain the following Corollary.

Corollary 2.16 ([2]). Let $\mathrm{f}$ given by (1.2) be in the class $\mathcal{S}_{\Sigma}^{*}(\beta)$, then

$$
\left|\mathrm{a}_{2}\right| \leqslant \sqrt{2(1-\beta)} \text {, and }\left|\mathrm{a}_{3}\right| \leqslant 4(1-\beta)^{2}+(1-\beta) .
$$

\section{Acknowledgment}

This work is supported by UKM grant: GUP-2017-064.

\section{References}

[1] R. M. Ali, S. K. Lee, V. Ravichandran, S. Supramaniam, Coefficient estimates for bi-univalent Ma-Minda starlike and convex functions, Appl. Math. Lett., 25 (2012), 344-351. 1

[2] Ş. Altinkaya, S. Yalçin, Coefficient bounds for certain subclasses of $m$-fold symmetric bi-univalentf Functions, J. Math., 2015 (2015), 5 pages. iv, 2.7, 2.8, 2.15, 2.16

[3] M. K. Aouf, R. M. El-Ashwah, A. M. Abd-Eltawab, New subclasses of bi-univalent functions involving Dziok-Srivastava operator, ISRN Math. Anal., 2013 (2013), 5 pages. 1

[4] D. A. Brannan, J. G. Clunie, Aspects of Contemporary Complex Analysis, Academic Press, New York, (1980).

[5] D. A. Brannan, T. S. Taha, On some classes of bi-univalent functions, Studia Univ. Babe-Bolyai Math., 31 (1986), $70-77$. $\mathrm{V}, \mathrm{V}$

[6] S. Bulut, Coefficient estimates for initial Taylor-Maclaurin coefficients for a subclass of analytic and bi-univalent functions defined by Al-Oboudi differential operator, Sci. World J., 2013 (2013), 6 pages.

[7] S. Bulut, Coefficient estimates for new subclasses of analytic and bi-univalent functions defined by Al-Oboudi differential operator, J. Funct. Spaces Appl., 2013 (2013), 7 pages.

[8] S. Bulut, Coefficient estimates for a class of analytic and bi-univalent functions, Novi Sad J. Math., 43 (2013), 59-65.

[9] S. Bulut, Coefficient estimates for general subclasses of $\mathrm{m}$-fold symmetric analytic bi-univalent functions, Turk. J. Math., 40 (2016), 1386-1397.

[10] S. Bulut, Coefficient estimates for a new subclass of analytic and bi-univalent functions, An. Ştiint. Univ. Al. I. Cuza Iaşi. Mat. (N.S.), 62 (2016), 305-311.

[11] M. Çağlar, H. Orhan, N. Yağmur, Coefficient bounds for new subclasses of bi-univalent functions, Filomat, 27 (2013), 1165-1171.

[12] E. Deniz, Certain subclasses of bi-univalent functions satisfying subordinate conditions, J. Class. Anal., 2 (2013), 49-60.

[13] E. Deniz, M. Çağlar, H. Orhan, Second Hankel determinant for bi-starlike and bi-convex functions of order $\beta$, Appl. Math. Comput., 271 (2015), 301-307. 1

[14] P. L. Duren, Univalent Functions, Springer-Verlag, New York, (1983). 1.5

[15] B. A. Frasin, M. K. Aouf, New subclasses of bi-univalent functions, Appl. Math. Lett., 24 (2011), 1569-1573. 1 
[16] S. P. Goyal, P. Goswami, Estimate for initial Maclaurin coefficients of bi-univalent functions for a class defined by fractional derivatives, J. Egyptian Math. Soc., 20 (2012), 179-182. 1

[17] S. G. Hamidi, J. M. Jahangiri, Unpredictably of the coefficients of m-fold symmetric bi-Starlike functions, Inter. J. Math., 2014 (2014), 8 pages. iv

[18] T. Hayami, S. Owa, Coefficient bounds for bi-univalent functions, PanAmer. Math. J., 22 (2012), 15-26. 1

[19] W. Koepf, Coefficient of symmetric functions of bounded boundary rotations, Proc. Amer. Math. Soc., 105 (1989), $324-$ 329. 1

[20] M. Lewin, On a coefficient problem for bi-univalent functions, Proc. Amer. Math. Soc., 18 (1967), 63-68. 1

[21] G. Murugusundaramoorthy, N. Magesh, V. Prameela, Coefficient bounds for certain subclasses of bi-univalent function, Abstr. Appl. Anal., 2013 (2013), 3 pages. 1

[22] E. Netanyahu, The minimal distance of the image boundary from the origin and the second coefficient of a univalent function in $|z|<1$, Arch. Ration. Mech. Anal., 32 (1969), 100-112.

[23] C. Pommerenke, Univalent Functions, Vandenhoeck \& Ruprecht, Göttingen, (1975).

[24] S. Porwal, M. Darus, On a new subclass of bi-univalent functions, J. Egyptian Math. Soc., 21 (2013), 190-193.

[25] H. M. Srivastava, S. Bulut, M. Çağlar, N. Yağmur, Coefficient estimates for a general subclass of analytic and bi- univalent functions, Filomat, 27 (2013), 831-842. 1

[26] H. M. Srivastava, A. K. Mishra, P. Gochhayat, Certain subclasses of analytic and bi-univalent functions, Appl. Math. Lett., 23 (2010), 1188-1192. 1, vii, vii, 2.6, 2.14

[27] H. M. Srivastava, S. Sivasubramanian, R. Sivakumar, Initial coefficient bounds for a subclass of m-fold symmetric bi-univalent functions, Tbilisi Math. J., 7 (2014), 1-10. vi, vi, 2.5, 2.13

[28] S. Sümer Eker, Coefficient bounds for subclasses of m-fold symmetric bi-univalent functions, Turkish J. Math., 40 (2016), 641-646. 1

[29] T. S. Taha, Topics in univalent function theory, Ph.D. thesis, University of London, UK, (1981).

[30] Q.-H. Xu, Y.-C. Gui, H. M. Srivastava, Coefficient estimates for a certain subclass of analytic and bi-univalent functions, Appl. Math. Lett., 25 (2012), 990-994.

[31] Q.-H. Xu, H.-G. Xiao, H. M. Srivastava, A certain general subclass of analytic and bi-univalent functions and associated coefficient estimate problems, Appl. Math. Comput., 218 (2012), 11461-11465. 1 